\title{
Effect of Decompression Therapy Combined with Joint Mobilization on Patients with Lumbar Herniated Nucleus Pulposus
}

\author{
Younghwa Lee, PhD, $\mathrm{PT}^{1}$, , Chang-Ryeol Lee, PhD, PT ${ }^{2)}$, Misuk Cho, PhD, $\mathrm{PT}^{2}$ ) \\ 1) Center of Physical Therapy, Kimjun's Orthopaedic Clinic \\ 2) Department of Physical Therapy, Korea Nazarene University: Wolbong Ro 48, Seobuk-gu, Cheonan- \\ Si, Chungcheongnam-do, 330-718 Republic of Korea. TEL: +82 41-570-4167, FAX: +82 41-570-7925, \\ E-mail:crlee@kornu.ac.kr
}

\begin{abstract}
Purpose] The purpose of this study was to examine the effects of decompression therapy combined with joint mobilization on the pain and range of motion of patients with lumbar herniated nucleus pulposus. [Subjects] A total of 31 subjects were randomly assigned to an experimental group of 17 subjects and a control group of 14 subjects. [Methods] The experimental group received joint mobilization and decompression therapy, while the control group received thermotherapy, electrotherapy, and decompression therapy. Pain on visual analog scale (VAS) and the ranges of motion of flexion, extension, and lateroflexion were measured in both groups before the treatment and after the four weeks of treatment. [Results] Comparison of visual analog scale scores and the ranges of motion before and after treatment showed greater statistically significant differences in the experimental group than in the control group. [Conclusion] Decompression therapy combined with joint mobilization was effective as an intervention method for relieving pain and increasing the range of motion of the lumbar spine in patients with lumbar herniated nucleus pulposus.

Key words: Decompression, Lumbar HNP, Mobilization
\end{abstract}

(This article was submitted Mar. 21, 2012, and was accepted Apr. 26, 2012)

\section{INTRODUCTION}

Lumbar herniated nucleus pulposus is one of the causes of low back pain and describes the condition in which rupture of the annulus fibrosis causes the nucleus pulposus to compress the dura mater or neuromuscles in the spinal cord. Representative symptoms of this disease include lumbosacral pain, radiating pain caused by the neuromuscles, weakened muscle strength, paresthesia, and limited ranges of motion of the spine ${ }^{1)}$. Most cases are treated with conservative treatments such as physical therapy, exercise therapy, drug therapy, injections, etc., but cases of cauda equina syndrome and those that show progressive motor nerve damage and unbearable pain are treated surgically ${ }^{2}$. One effective conservative treatment is axial decompression therapy, which reduces interdiscal pressure and relieves low back pain and symptoms of herniated nucleus pulposus ${ }^{3)}$. Similarly, joint mobilization increases the range of motion of the spine and reduces pain in the spine ${ }^{4}$. Some studies of decompression therapy and of joint mobilization have been reported, but studies that have examined the effectiveness of a combination of these two treatments are rare, especially in cases of nucleus pulposus.

Therefore, the aim of this study was to examine the effectiveness of a combined decompression therapy and joint mobilization treatment on pain relief and increased range of motion in patients with lumbar herniated nucleus pulposus.

\section{SUBJECTS AND METHODS}

The subjects of this study were 31 patients who visited $\mathrm{K}$ orthopedic clinic in Daegu, Korea between August 2011 and February 2012, and who were diagnosed with lumbar herniated nucleus pulposus based on the results of magnetic resonance imaging (MRI). The 31 subjects were randomly assigned either to the experimental group, 17 subjects, or to the control group, 14 subjects. The experimental and control groups did not differ significantly with respect to sex, age, or weight $(\mathrm{p}>0.05)$.

Study inclusion criteria were: patients with L4-5 or L5-S1 lumbar herniated nucleus pulposus, lumbosacral and lower extremity radiating pain, and a limited range of motion of the lumbar spine. Exclusion criteria were: patients with compression fractures, spondylolisthesis, spinal stenosis, degenerative spondylitis having bony spurs, or previous back surgery. All subjects were selected from those who voluntarily participated after receiving an oral explanation about the study and who submitted a signed consent form. Both groups were treated for four weeks. The treatments were provided 3-4 times a week and the total number of treatments provided to each subject was between 12 and 15. Both groups were first treated with a SpineMed (CERT Healthsciences, LLC, Baltimore, USA), which is equipment that enables axial decompression therapy. The subject assumed a supine position on the traction table, then knee 
support was applied under the subject's knees, and the subject's pelvis was fixed on both sides at the regions of the anterior superior iliac spine using a pelvis-fixing device. Traction power, which is the intensity of the treatment, was set initially to $30 \%$ of the weight of the subject and then increased to $40 \%$ in the second week and $50 \%$ in the third or fourth week. The traction power was reduced or maintained when pain occurred due to increased traction power. The treatment time was 20 minutes and the ratio between hold time and rest time was set to $2: 1$, so a hold time of $60 \mathrm{~s}$ was followed by a rest time of 30 seconds and this sequence was repeated; traction during the rest time was reduced to $50 \%$ of that during the hold time.

After decompression therapy, the experimental group underwent joint mobilization treatment for 15 minutes on a table for manual therapy. Class 1 vibration therapeutics were used for joint mobilization of hyper mobile segments between L4-5 and L5-S1, where lumbar herniated nucleus pulposus occurred, in order to relieve pain. Class 3 vibration therapeutics were used in hypo mobile segments between $\mathrm{T} 12$ and L4 to increase the range of motion ${ }^{5}$. Flexion therapeutic mobilization was performed with the patient in a lateral recumbent position on the manual bed. The hip joints and knee joints on both sides of the patient were maximally flexed and the therapist stood at the ventral side of the patient. The therapist placed his fixing hand on the spinous process of the upper lumbar spine of the segment to be treated, and placed his other hand for performing mobilization on the spinous process of the lower lumbar spine of the segment to be treated, than brought his trunk into tight contact with the two knee joints of the patient. The therapist then moved the hand that fixed the spinous process of the upper lumbar spine, in the caudal-ventral direction while maintaining tight trunk contact with the patients knees to perform therapeutic mobilization flexing the segment being treated. A towel was inserted between the lumbar spine and the bed so that the lumbar spine was in the neutral position and the upper lumbar spine was fixed so that it would not move.

Extension therapeutic mobilization was performed with the patient in a lateral recumbent position on the manual bed. The hip joints and knee joints on both sides of the patient were flexed to $90^{\circ}$ and the therapist stood on the ventral side of the patient. The therapist again placed one hand on the spinous process of the upper lumbar spine of the segment to be treated, and his other hand on the spinous process of the lower lumbar spine of the segment to be treated, and then brought his trunk into tight contact with the two knee joints of the patient. The therapist then moved the hand that fixed the spinous process of the upper lumbar spine, in the cranial-dorsal direction while maintaining tight trunk contact with the patients knees to perform therapeutic mobilization extending the segment being treated. A towel was again inserted between the lumbar spine and bed so that the lumbar spine was in the neutral position and care was taken to prevent change in the angle between the hip joint and knee joint.

Lateroflexion therapeutic mobilization was performed with the patient in a lateral recumbent position on the manual bed. The hip joints and knee joints on both sides of the patient were flexed to $90^{\circ}$ and the therapist stood on the ventral side of the patient. At this time, the patient's lumbar spine was slightly flexed. The therapist placed the fixing hand on the spinous process of the lower lumbar spine of the segment to be treated and brought his trunk into tight contact with the pelvis and femoral region of the patient to fix the region. The therapist fixed the lower thorax of the patient with his forearm and placed his hand for performing mobilization on the spinous process of the upper lumbar spine of the segment to be treated. The therapist then used coupling movements to turn the lower thorax and upper lumbar spine of the patient so that lateroflexion would occur in the segment to be treated. At this time, the patient's trunk was raised using a manual bed or pillow to adjust lateroflexion, to facilitate lateroflexion in the patient's lumbar spine.

The control group received conventional physical therapy following decompression therapy. Conventional treatments included the use of hot pack compresses for 10 minutes and electrotherapy using interference waves for 10 minutes. Pain on visual analog scale (VAS) and range of motion of lumbar spine flexion, extension, and lateroflexion using a 3D motion analyzer were measured in both groups before the treatment and after the four weeks of treatment. Each patient's subjective pain was determined by having the patients indicate their degree of pain using a VAS scale consisting of a thick $10 \mathrm{~cm}$ long line. The ranges of motion of lumbar spine flexion, extension, and lateroflexion were measured using a three-dimensional motion analyzer composed of a PC, active markers that send ultrasonic signals, a main body, and cable adaptors. The distance between the measurement sensor and the patient was maintained at around $80 \mathrm{~cm}$ and the measurement sensor was maintained in a horizontal position. Two markers made for spine measurement were used as ultrasonic markers. One marker was fixed with Velcro to the top of the sacrum, facing upward, for use as the reference marker. The second marker was fixed with Velcro to the bottom of the thoracic spine, again facing upward, for use as the measuring marker. The 3D positions of each marker, obtained through the measurement sensor, were converted into coordinates using the PC Windows program Winspine 1.71 (Zebis Medizintechnik, Gmbh, Isny, Germany) and angles between individual coordinates were measured.

The results of this study were analyzed using SPSS 12.0 KO (SPSS, Chicago, IL, USA). The paired t-test was used conducted to compare the VAS and the ranges of motion of flexion, extension, and lateroflexion in the experimental group and the control group before the treatment and four weeks after the treatment. ANCOVA was conducted to determine which group received the more effective treatment. The statistical significance level was chosen as 0.05 .

\section{RESULTS}

The experimental group showed a statistically significant decrease in VAS score and significant increases in the ranges of motion of flexion, extension, and lateroflexion $(p<0.05)$. The control group showed a statistically significant decrease 
Table 1. Comparison of VAS and range of motion in the Experimental and Control groups

\begin{tabular}{lcccc}
\hline \multirow{2}{*}{ Variables } & \multicolumn{2}{c}{ Experimental group $(\mathrm{n}=17)$} & \multicolumn{2}{c}{ Control group $(\mathrm{n}=14)$} \\
\cline { 2 - 5 } & \multicolumn{1}{c}{ Before } & After 4 wk & Before & After 4 wk \\
\hline VAS** & $5.29 \pm 1.45$ & $2.29 \pm 1.11^{*}$ & $4.57 \pm 1.34$ & $3.29 \pm 0.61^{*}$ \\
Flexion** & $37.53 \pm 11.90$ & $44.71 \pm 9.03^{*}$ & $38.93 \pm 8.01$ & $41.66 \pm 7.67^{*}$ \\
Extension** & $9.65 \pm 4.12$ & $13.82 \pm 3.40^{*}$ & $10.05 \pm 3.78$ & $12.36 \pm 4.48^{*}$ \\
RLF** & $17.35 \pm 4.64$ & $21.76 \pm 4.97^{*}$ & $19.36 \pm 3.37$ & $20.50 \pm 2.85$ \\
LLF** $^{*}$ & $18.53 \pm 4.78$ & $22.12 \pm 4.00^{*}$ & $18.64 \pm 3.39$ & $19.71 \pm 2.64$ \\
\hline
\end{tabular}

(unit: ${ }^{\circ}$ ) Mean $\pm \mathrm{SD}, * \mathrm{p}<0.05$ : paired t-test, ${ }^{* *} \mathrm{p}<0.05$ : ANCOVA test, VAS: visual analog scale, RLF: right lateral flexion, LLF: left lateral flexion.

in VAS score and significant increases in the ranges of motion of flexion and extension $(\mathrm{p}<0.05)$. In the comparison of the experimental group and control group results, the experimental treatment was found to be more effective at reducing VAS score and improving the ranges of motion of flexion, extension, and lateroflexion $(\mathrm{p}<0.05)$ (Table 1).

\section{DISCUSSION}

The aim of this study was to examine the effects of combining decompression therapy with joint mobilization on pain relief and improvement in the range of motion of patients with L4-5 or L5-S1 lumbar herniated nucleus pulposus. Lumbar herniated nucleus pulposus occurs most frequently in the lumbar spine and typically responds poorly to conventional traction treatment because insufficient traction power is delivered to the lumbar spine due to the contraction of the paravertebral muscles in a protective reflex $^{6}$. Therefore, facilities that enable axial decompression therapy and that can produce sufficient traction power have recently been developed and utilized in clinics, and they have been reported to be effective for pain relief ${ }^{7}$.

A previous study examined the effects of decompression therapy in 778 patients with herniated discs, degenerated discs, or facet syndrome and reported significant pain relief and increases in mobility ${ }^{3}$. Similarly, Ramos and Martin $^{8)}$ reported that decompression therapy was effective at relieving pain in patients with lumbar herniated nucleus pulposus who were not indicated for surgery. Another recent study conducted decompression therapy combined with joint mobilization and lumbar spine stabilizing exercise for 30 dicogenic low back pain patients, and reported significant improvements in the Oswestry Disability Index (ODI) and the straight leg raise $(\mathrm{SLR})^{9)}$. These findings may have been the result of decreases in inflammatory exudates, since the negative pressure generated in the disc by decompression therapy may have created diffusion gradients, resulting in improved solute transport ${ }^{8)}$. Blood flow from the regions around the pyramidal endplate and epidural blood vessels may be increased the supply of body fluids and nutrients necessary for the recovery of intervertebral discs and may also have increased the diameter of intervertebral foramens, leading to increased blood flow in vertebral nerves and intervertebral foramens. Decreases in mechanical compression that was causing neurologic inflammation may also have contributed to the observed effects ${ }^{10)}$

Joint mobilization has primarily been used to relieve pain and increase the range of motion in vertebrae with dysfunction of vertebral joints ${ }^{4)}$. Bronfort ${ }^{11)}$ indicated that joint mobilization was more effective at increasing the range of motion of lumbar spine flexion and extension in 26 lower back pain patients than were other physical therapies and reported significant increases in the ranges of motion ${ }^{12)}$. The pain relief observed in these previous studies may have occurred because the joint mobilization generated by class 1 vibration therapeutics triggered the firing of the mechanical sensory receptors in the joint capsule, the spindle receptors in muscle fibers, and the mechanical receptors in Golgi tendon organs. The resulting action potential generated by these primary myelinated large diameter afferent fibers would then be delivered to the hind leg region of the vertebrae to stimulate inhibitory interneurons, thereby directly reducing the action potential of small diameter unmyelinated axons and myelinated axons ${ }^{13}$. The increases in the range of motion of the lumbar spine may have occurred because the joint mobilization generated by class 3 vibration therapeutics extended the tissues around the joints or joint capsules and increased the range of motion ${ }^{14)}$.

The results of the present study, in which joint mobilization was combined with decompression therapy in patients with lumbar herniated nucleus pulposus, show significant pain relief and increases in the ranges of flexion, extension, and lateroflexion as observed in previous studies. However, the previous studies examined decompression therapy and joint mobilization separately, not as combined treatments, and reported that joint mobilization was effective at increasing the range of motion of low back pain patients in almost all cases. The present study extended these findings to show that positive effects of the combined treatment were found in the hypo-mobile segments found in patients, who also had hyper-mobile segments, with lumbar herniated nucleus pulposus.

In conclusion, a combined treatment of decompression therapy and joint mobilization is an effective intervention for relieving pain and increasing the range of motion of patients with herniated nucleus pulposus. These findings should be validated through further studies of patients with lumbar herniated nucleus pulposus. 


\section{ACKNOWLEDGEMENT}

This study was financially supported by the research fund of Korea Nazarene University in 2012.

\section{REFERENCES}

1) Park JT, Park YK: Manual of clinical orthopedics. Seoul: Hyunmoon press, 2002, pp 319-325.

2) Postacchini F: Results of surgery compared with conservative management for lumbar disc herniations. Spine, 1996, 21: 1383-1387. [Medline] [CrossRef]

3) Gose EE, Naguszewski WK, Naguszewski RK: Vertebral axial decompression therapy for pain associated with herniated or degenerated disc or facet syndrome: an outcome study. Neurol Res, 1998, 20: 186-190. [Medline]

4) Mennell JM: The validation of the diagnosis "joint dysfunction" in the synovial joints of the cervical spine. J Manipulative Physiol Ther, 1990, 13: 7-12. [Medline]

5) Kaltenborn FM: The spine: Basic evaluation and mobilization techniques Minneapolis: Orthopedic Physical Therapy Products press, 2001, pp148 162.
6) Tekeoglu I, Adak B, Bozkurt M, et al.: Distraction of lumbar vertebrae in gravitational traction. Spine, 1998, 23: 1061-1063. [Medline] [CrossRef]

7) Beattie PF, Nelson RM, Michener LA, et al.: Outcomes after a prone lumbar traction protocol for patients with activity-limiting low back pain. Arch Phys Med Rehabil, 2008, 89: 269-274. [Medline] [CrossRef]

8) Ramos G, Martin W: Effects of vertebral axial decompression on intradiscal pressure. J Neurosurg, 1994, 81: 350-353. [Medline] [CrossRef]

9) Ma SY, Kim HD: The efficacy of spinal decompression via DRX3000 combined with a spinal mobilization and a lumbar stabilization exercise program for patients with discogenic low back pain. J Phys Ther Sci, 2010, 22: 345-354. [CrossRef]

10) Onel D, Tuzlaci M, Sari H, et al.: Computed tomographic investigation of the effect of traction on lumbar disc herniations. Spine, 1989, 14: 82-90. [Medline] [CrossRef]

11) Bronfort G, Haas M, Evans R, et al.: Efficacy of spinal manipulation and mobilisation for low back pain and neck pain. A systematic review and best evidence synthesis. Spine, 2004, 4: 335-356. [CrossRef]

12) Konstantinou K, Foster N, Rushton A, et al.: Flexion mobilizations with movement techniques: the immediate effects on range of movement and pain in subjects with low back pain. J Manipulative Physiol Ther, 2007, 30: 178-185. [Medline] [CrossRef]

13) The Korean Orthopaedic Association: ORTHOPAEDICS. Seoul: Newest Medicine Company press, 2006, pp 1153-1171.

14) Kisner C, Colby LA: Therapeutic Exercise: Foundations and Techniques. Philadelphia: F.A. Davis press, 2002, pp 218-276. 